\title{
Can Learned Experiences Accelerate the Implementation of Sustainable Development Goal 11? A Framework to Evaluate the Contributions of Local Sustainable Initiatives to delivery SDG 11 in Brazilian Municipalities
}

\author{
Ana C L Almeida ${ }^{\mathrm{i}^{*}}$, James C R Smart ${ }^{\mathrm{i}}$, Peter Davey ${ }^{\mathrm{i}}$
}

\begin{abstract}
The adoption of Sustainable Development Goals (SDGs) by the UN General Assembly in September 2015, particularly SDG 11, reiterated the importance of sustainable planning in cities. This study evaluates the contribution of four pre-existing major local planning initiatives towards increasing the capacity of Brazilian cities to deliver sustainable development. A conceptual framework was designed to evaluate four dimensions of sustainable development: social, environmental, economic and political. This evaluation draws lessons to develop an action framework to facilitate the implementation of SDG 11 at the local city level. The evaluation of planning initiatives in 15 Brazilian municipalities was based on a mixed methods and triangulation approach and seems to be helpful for enhancing and facilitating implementation of SDG 11 in Brazilian municipalities. In contrast, due to lack of systematic data from Brazilian municipalities it will be necessary to foster the development of adequate indicators to evaluate the effectiveness of SDG 11. Despite this, SDG 11 can become a referential goal to enhance sustainability in some cities and create opportunity to influence the Brazilian municipalities about the importance of sustainable planning.
\end{abstract}

Keywords: sustainable planning in cities; NVivo

\section{Introduction}

The 2030 Agenda on Sustainable Development adopted by the UN General Assembly in $2015^{1}$ recognizes the importance of robust planning strategies for overall implementation of the Agenda, and suggests that delivery of the Sustainable Development Goals (SDGs) should be built on existing planning instruments. Of the 17 SDGs, Goal 11 promotes the development of inclusive and sustainable cities through planning and management. This goal is the lynchpin of local implementation of the SDGs (UNDP, 2016). This will inevitably place considerable additional demands on local governments whose resources are already over-stretched by implementing existing local-level development and sustainability initiatives. To-date very few effective linkages have been identified between SDG11 and existing local-level policies for sustainable development, further increasing the challenge posed by SDG11 implementation

1 A/RES/70/1 - Resolution adopted by General Assembly on 25 September 2015: 'Transforming our world: the 2030 Agenda for Sustainable Development'. 
(Satterthwaite, 2016; Patel et al. 2017; Corbett \& Mellouli, 2017; Arslan et al. 2016). Since commencement of the SDG process in 2015, the challenge for governments at every level has been: 'How to recognise and incorporate links and trade-offs across all 17 SDG Goals effectively within national planning processes and strategies?' (Hall et al. 2017). In fact, although existing planning instruments could potentially accelerate the implementation of SDG 11, it remains unclear how past lessons can best be integrated to strengthen local sustainable agendas and SDG 11 implementation. This research evaluates the performance of previous local-scale urban sustainable development initiatives and draws on the lessons learned to produce a framework that can potentially enhance effective implementation of SDG11.

In Brazil, four major initiatives that seek to promote sustainability in cities have been implemented since the mid-1990s: the Healthy Municipalities, Cities and Communities (HMC) initiative, Local Agenda 21 (LA21), the Millennium Development Goals (MDGs), and Master Plans (MPs). All these initiatives are based on planning models designed in principle to assist municipalities to develop more structured local planning (Lichfield et al. 1975; WHO, 2000; UN, 2012; MCidades, 2004; SG-PR, 2009; Green, 2012).

The general strategy employed by these local planning initiatives was to encourage intersectorial and participatory action by all stakeholders, aiming to improve and transform social and environmental conditions. By 2014 these initiatives had already been implemented by Brazilian Municipalities in ways that were intended to have a positive influence on sustainable development at a local level.

The existing guidelines place considerable emphasis on participatory diagnosis to build public commitment and promote community participation. However, none of these guidelines emphasise the importance of integrated and systematic evaluation, or indicate how this evaluation should be conducted by municipalities. This is a major gap that restricts opportunities for identifying barriers and enablers of successful implementation, and prevents lessons learned from being carried forward to implementation of future initiatives. As a consequence, although it has been more than 20 years since planning initiatives were first implemented in Brazil, the lessons from HMC, LA21, MDGs and MPs (applied individually or in combination) have not been identified clearly in terms of effective delivery of sustainable development at a local level.

In response to the lack of integrative approaches to evaluate the success, or otherwise, of local-level urban sustainable development initiatives in Brazil, the first objective of this study is to develop an evaluation framework to assess the implementation and effectiveness of the four existing planning initiatives, namely HMC, LA21, MDGs, and the MPs in combination. Drawing on findings from applying this evaluation framework to existing planning initiatives in a sample of 15 Brazilian municipalities, the second objective of this study is to identify key success factors and barriers that may facilitate, or inhibit, future implementation of SDG 11 at the local level. The study concludes by drawing these lessons together to produce an action framework to assist future implementation of SDG 11 at a local level.

\section{Research Methodology}

\subsection{Selection of Municipalities}

More than a thousand Brazilian Municipalities developed local planning 
initiatives to promote sustainable development via HMC, LA21, the MDGs and/or MPs. Given this scale of implementation, implementation of HMC was chosen as the basis to limit the scope of study sample of municipalities addressed in this research. Implementation of the HMC initiatives is concentrated in Sao Paulo State and the current pattern of distribution within the State is further concentrated in the Campinas mesoregion $^{2}$ which contains 49 municipalities. For reasons of tractability, this study evaluated implementation performance of the four sustainability initiatives within a sample of 15 municipalities (30\%) in the Campinas mesoregion.

\subsection{Data Collection}

A new framework for evaluating the impact of implementing HMC, LA21, MDGs, and MPs initiatives in combination was therefore developed and applied across the 15 municipality study sample. Both quantitative and qualitative data were collected through an online survey and semi-structured interviews. The interviews were carried out after the survey and used to develop a more nuanced, higher level understanding of implementation processes, outcomes and effectiveness, and factors affecting the same, at the local level. An invitation to participate in an online survey was sent by email to 35 potential key informants (KI), including local government and civil society representatives, from February 2017 to April 2017. A total of 19 respondents agreed to participate in the online survey. Only two respondents were representatives of nongovernmental organization (NGOs) and no responses were received from community representatives. Participation in the survey by city size is reported in Table 2.

Table 2: Number of respondents and evaluated initiatives by city size.

\begin{tabular}{|c|c|c|c|}
\hline \multirow[t]{2}{*}{ City Size } & \multicolumn{2}{|c|}{ Key Informants (KI) } & \multirow{2}{*}{$\begin{array}{l}\text { Initiatives } \\
\text { evaluated in } \\
\text { combination }\end{array}$} \\
\hline & $\begin{array}{c}\text { Local } \\
\text { government }\end{array}$ & NGOs & \\
\hline Small & 5 & 0 & 10 \\
\hline Medium & 6 & 0 & 12 \\
\hline Big & 6 & 2 & 12 \\
\hline Total & 17 & 2 & 34 \\
\hline
\end{tabular}

Survey contacts also provided a basis for subsequent semi-structured interviews to increase the reliability and representativeness of sampling. These semi-structured interviews were carried out by telephone or in person with a further 15 key informants including experts, local government, and civil society representatives. Among the civil society group, there were representatives from NGOs and the business sector.

\subsection{Conceptual Evaluation Framework}

A conceptual framework was devised to evaluate whether more cohesive, integrated planning and implementation of local-level initiatives could potentially increase the effectiveness of development delivery at a local level. As shown in figure 1,

2 Mesoregion is a subdivision of Brazilian states, which collects municipalities with economic and social similarities. The term was created by the Brazilian Institute of Geography and Statistics - IBGE and is used for statistical purposes. Therefore, a Mesoregion is not a political or administrative unit. In Brazil, there are a total of 137 mesoregions within 27 states. 
distinct aspects of local development-related policies were disaggregated into four development dimensions: social, environmental, economic and political.
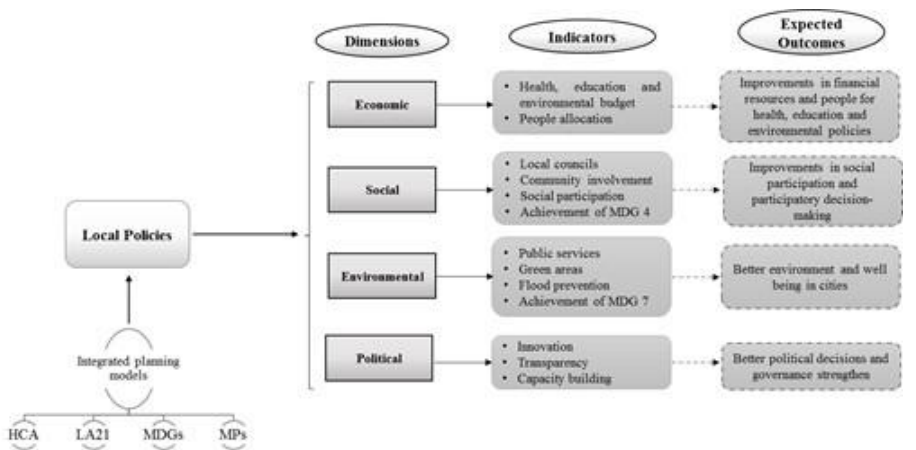

Figure 1: Conceptual

Framework for the evaluation of local planning initiatives.

For each dimension, a set of indicators was identified comprising distinct features of sustainability and expected outcomes. The indicators chosen make use of available statistical data from municipalities to map out the capacity of cities to deliver sustainable development. It was not the intention of this study to propose new indicators, but rather to use and enhance existing ones by collating them into a cohesive framework.

The conceptual framework was used to construct the online survey that collected quantitative and qualitative data simultaneously. The first part of the online survey was designed to collect general information on initiatives. Respondents were asked to assess the current state of implementation of all relevant initiatives in their municipality. Also, the first part of the survey included 48 closed questions. Respondents provided Likert scale responses to assess the status of each indicator for each dimension of development in the conceptual evaluation framework (Figure 1). Likert scales were arranged from 5 (strongly agree) to 1 (strongly disagree). The second part of the survey comprised openended questions to collect qualitative data and to identify how respondents thought that learned experience could be applied to enhance delivery of SDG 11 at local levels.

\subsection{Data Analysis}

Quantitative analysis of these responses was undertaken in SPSS version 23.0 (IBM Corp, 2015). Responses from qualitative questions in the online survey and interviews were transcribed, organised into reports and analysed through QSR- Nvivo 11 software using constant comparison (Glaser \& Strauss, 1967). The coding process was developed based on an inductive approach (Strauss \& Corbin, 1998). Any elements of the key informants' answers and interview transcripts were coded into free nodes. Those nodes were then structured into tree nodes and grouped by themes (top-level nodes). All qualitative data sources were analysed in order to obtain deeper insight into implementation of local planning initiatives.

\section{Results}

\subsection{Quantitative Analysis}

Quantitative analysis was conducted to assess five different aspects: i) implementation of local initiatives; ii) the economic dimension of development and allocation of financial resources; iii) the social dimension of development and community 
participation; iv) the environmental dimension of development and local aspects of sustainability; v) the impact of combined implementation of development initiatives on municipal policies. The frequency distribution of responses across Likert-scale categories for all quantitative survey questions is shown in Table 3 , together with median scores.

Table 3: Results of Quantitative Analysis.

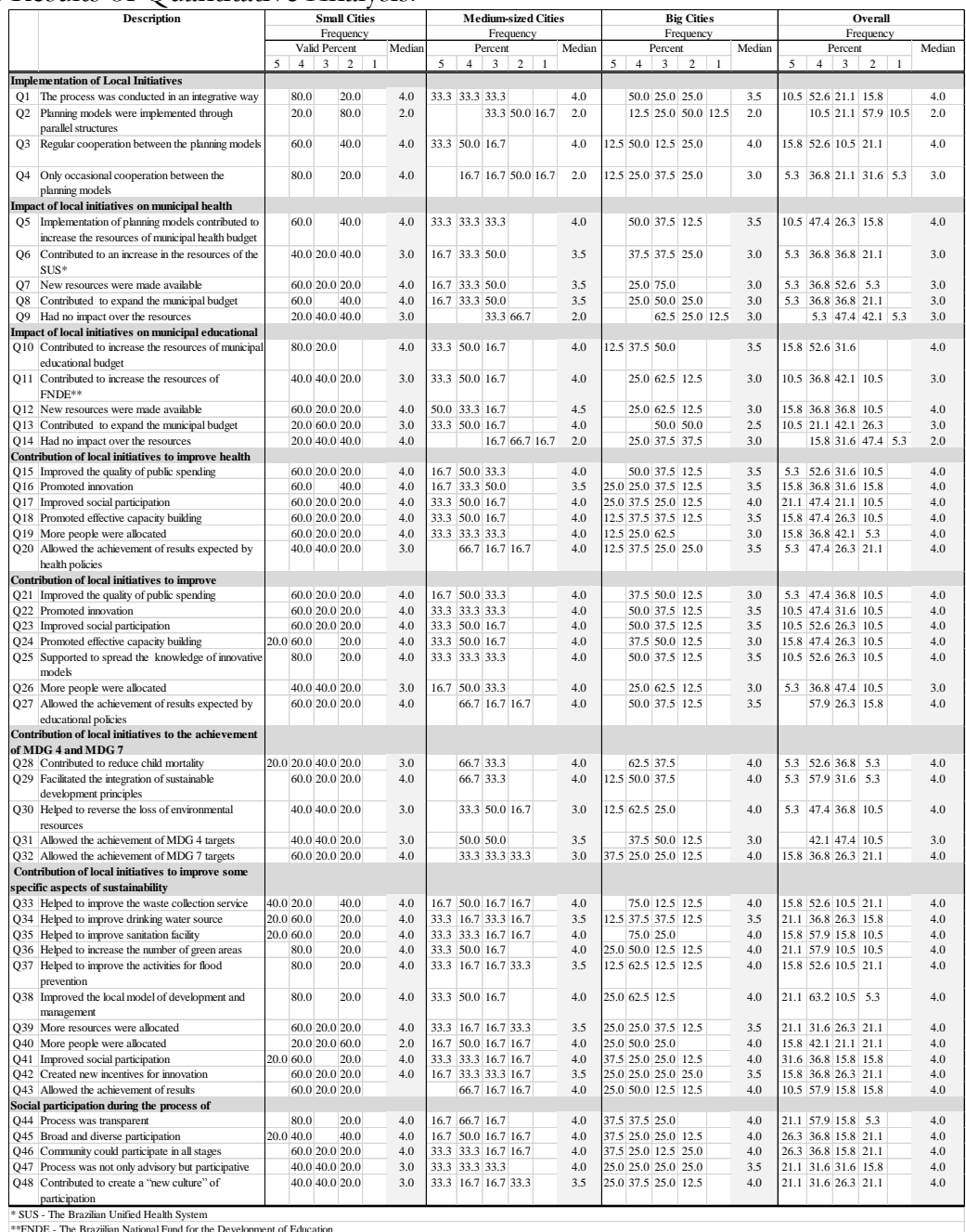

\subsubsection{Implementation of Local Initiatives}

The distribution of planning initiatives by implementation phase and city size is shown in figure 2. Based on the frequency distribution across the different implementation phases, it is clear that the state of progress differs depending on city size. The majority of all planning initiatives were reported to still be in the diagnostic or implementation phases. 
In small cities, no initiatives have so far been evaluated whereas a much higher proportion of initiatives have proceeded to the evaluation stage in big cities (42\%). Results also revealed that initiatives have only been officially interrupted in big cities, although this applied in only $8 \%$ of cases.

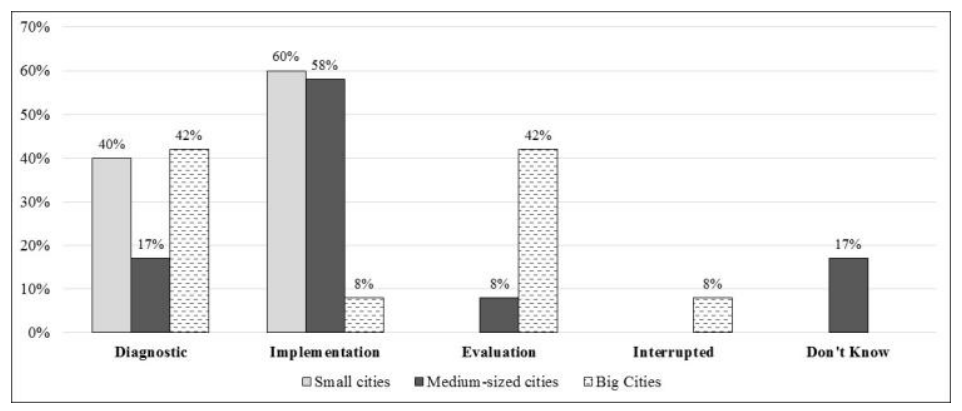

Figure 2: Phases of implementation of combined local planning initiatives (HMC, LA21, MDGs, and $M P s)$.

\subsubsection{Economic Dimension and Financial Resources}

The interaction between local initiatives and financial resources was evaluated from three perspectives: i) direct contribution to municipal budgets (Q5 and Q10); ii) indirect influence through external partnerships (Q7, Q12, and Q39); and iii) essential conditions without which budgetary expansion would not be possible (Q8 and Q13). Figure 3 shows responses separately for the educational, health and environmental sectors, segregated by city size. Overall, it can be seen that the perceived impacts of combined local initiatives on municipal budgets differed depending on city size. Based on the median response, it seems that combined local initiatives are perceived to have directly influenced health and educational budgets in all city sizes (Q5 and Q10), but in big cities only $50 \%$ of respondents considered that this was the case. Regarding external influences through partnerships, only in small cities were a majority of respondents convinced that local initiatives had positive effect on health, educational and environmental budgets (Q7, Q12, and Q39). In medium cities 50\% of respondents were unsure about impacts on health budgets, but the majority agreed that there had been a positive influence on educational budgets. Results were inconclusive for environmental budgets. In big cities, the majority of respondents don't know if combined local initiatives have influenced health and educational budgets, whereas equal numbers of respondents felt that combined local initiatives had had a positive impact, or were unconvinced about a positive impact, on the environmental budget. Finally, when asked if local initiatives were an essential condition for expanding municipal budgets (Q8 and Q13), the majority of participants from small cities agree that local initiatives were essential for increasing health budgets, but they were not sure about educational budgets. In medium cities, the majority of respondents agreed that local initiatives were essential for expanding the educational budget but could not say about health budget. In big cities, $50 \%$ of respondents were unsure about the impacts of local initiatives on both budgets, and $50 \%$ of big city respondents considered that initiatives had no impact on the educational budget. 


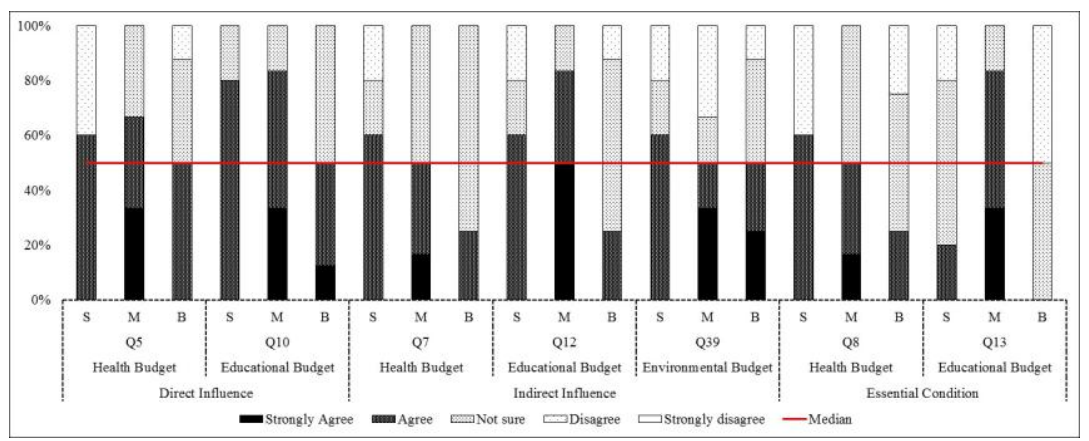

Figure 3: Responses in the stakeholder questionnaire to questions relating to the economic dimension of sustainable development. The proportion of respondents by Likert-scale response category is shown separately by city size ( $S=$ small city, $M=$ medium city, $B=$ big city).

\subsubsection{Social Dimension, Community Participation and Achievement of MDG 4}

Interaction between local initiatives and the social dimension of development was evaluated based on three indicators: i) the impact of local initiatives on the development of participatory health, educational and environmental policies (Q17, Q23, and Q41); ii) social participation in the process of implementing combined local initiatives (Q45 to Q48); and iii) the contribution of local initiatives to the achievement of MDG 4 (Q28 and Q31). Survey responses for outcomes relating to social participation are summarized in figure 4. Results indicate that local initiatives are considered to make a strong contribution towards improving social participation in health, education and environmental policies, regardless city size (Q17, Q23, and Q41).

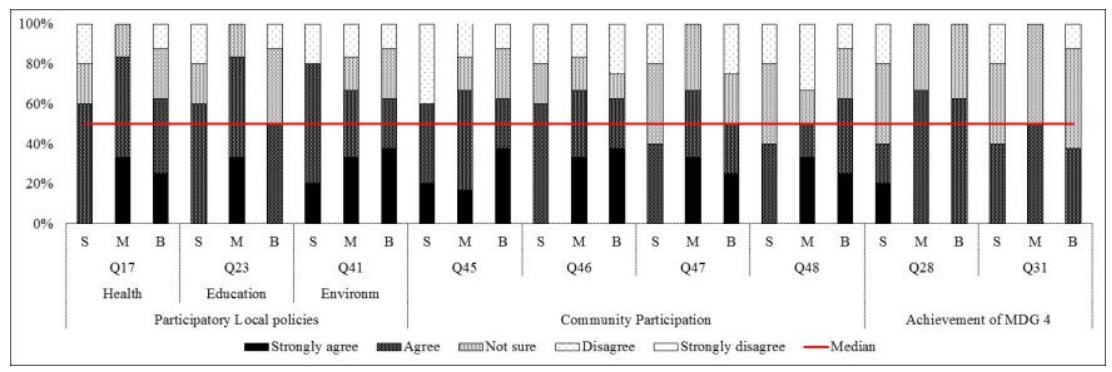

Figure 4: Responses in the stakeholder questionnaire to questions relating to the social dimension of sustainable development. The proportion of respondents who provided the median response is shown separately by city size ( $S=$ small city, $M=$ medium city, $B=$ big city).

Respondents' perceptions of the breadth and diversity of participation (Q45) and across all stages of the various initiatives (Q46) also indicate a positive perception of local the impact of initiatives on participatory processes, regardless city size.

On another level, results also reveal that some basic concerns about community participation remain, indicating that there is some scope for improving participatory processes. In small cities, respondents are not convinced whether participatory processes were merely advisory or whether they created a "new culture" of participation (Q47 and Q48). A majority of participants from medium cities agreed that the process was participative (67\%), but 33\% disagreed and 17\% were unsure whether a "new culture" of 
participation had emerged. In big cities, although $25 \%$ of respondents were not sure whether processes were participative, a majority $(63 \%)$ agreed that they contributed to a "new culture" of participation (Q48).

Regarding the achievement of the MDG 4 target to reduce the under-five mortality rate by twothirds, between 1990 and 2015, as shown in figure 4, only a minority of participants from small cities were convinced that combined local initiatives actually contributed to reducing child mortality (Q28) or achieving the MDG4 target. In contrast, clear majorities of respondents from medium and big cities (67\% and $63 \%$ respectively) agreed that local initiatives contributed to reducing child mortality (Q28). Nevertheless, when asked specifically about the influence of local initiatives on achieving the MDG 4 target, $50 \%$ of participants in both medium and big cities were unsure of any positive effect. This could perhaps be because they were not familiar with the MDGs.

\subsubsection{Environmental Dimension, Local Aspects of Sustainability and Achievement of MDG 7}

The evaluation was based on respondents' perceptions of some specific aspects of sustainability (Q29, Q30, and Q33 to Q38) and achievement of MDG 7 (Q32). As shown in figure 5 , the majority of respondents agreed that implementing the planning initiatives had led to improvements in almost all indicators of the environmental dimension of sustainable development (Q29, Q33, Q35, Q36, and Q38). The exceptions were with regard to reversing the loss of environmental resources (Q30), the quality of drinking water sources (Q34) and improving activities for flood prevention (for mediumsized cities). This suggests that local implementations of the planning initiatives had a very positive effect overall on environmental aspects of sustainable development at the local level.

Considering the contribution of local initiatives to the achievement of MDG 7 -Ensure environmental sustainability, even though this goal has not been achieved for Brazil as a whole, survey results reveal that the majority of respondents think that the local planning initiatives have definitely improved cities' ability to achieve MDG 7. This is not the case for respondents in medium-sized cities, however, where only $33 \%$ of respondents agree with this statement.

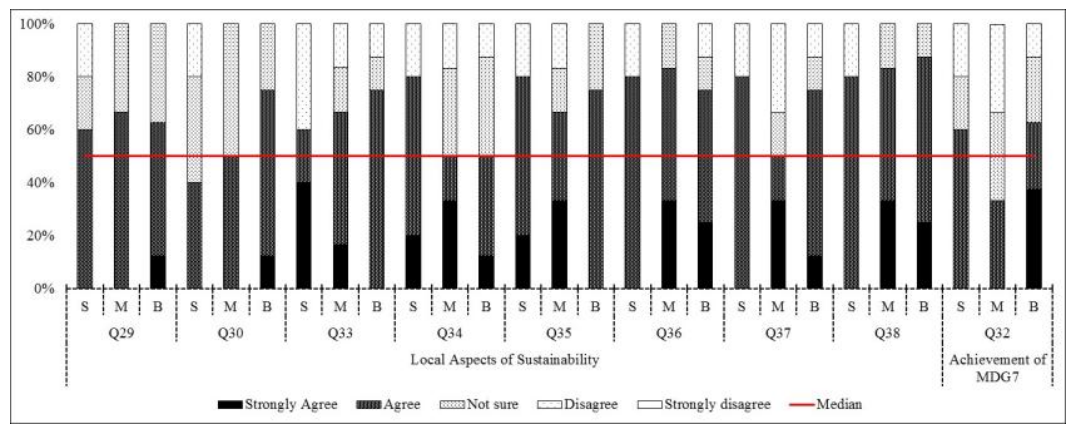

Figure 5: Responses in the stakeholder questionnaire to questions relating to the environmental dimension of sustainable development. The proportion of respondents who provided the median response is shown separately by city size ( $S=$ small city, $M=$ medium city, $B=$ big city). 


\subsubsection{Political Dimension}

Transparency (Q44), innovation in public policies (Q16, Q22, and Q42), and capacity building (Q18 and Q24) were the indicators used to track whether respondents felt that implementation of local planning initiatives improved the political dimensions of sustainable development. In terms of capacity building, survey results were mixed. Figure 6 shows that in small and medium-sized cities the majority of respondents agreed that the local planning initiatives promoted effective capacity building in health and education, but respondents in big cities were unsure whether the same could be said for their municipalities. With regard to whether the planning initiatives have engendered improved innovation in environment, health or education, the results were again mixed. An overwhelming majority $(79 \%)$ of respondents overall did, however, agree that local implementations of the planning initiatives had been transparent.

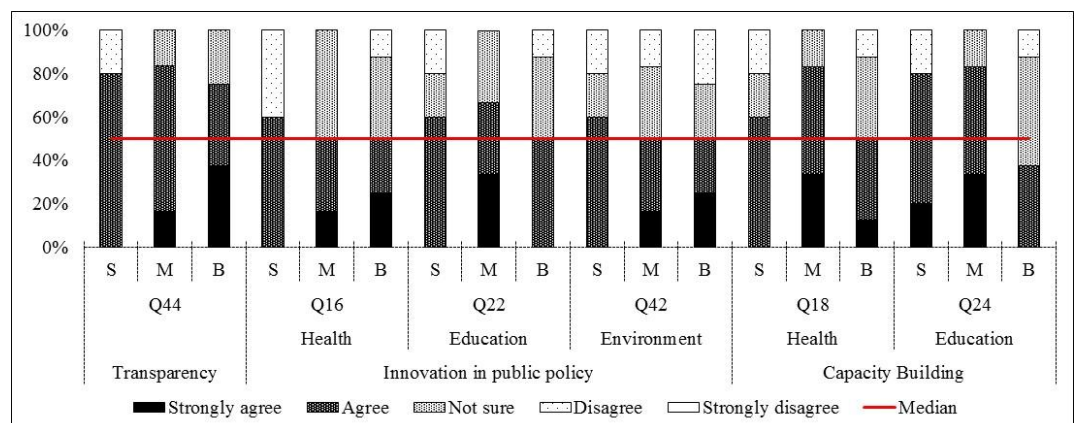

Figure 6: Survey responses for categorical variables relating to the political dimension of sustainable development, segregated by city size ( $S=$ small city, $M=$ medium city, $B=$ big city).

\subsection{Qualitative Analysis}

The results reflect the opinion of experts ${ }^{3}$, local government representatives and civil society organizations regarding the main barriers to integrated implementation of local initiatives. A total of eight main themes were considered to be the most important barriers by all categories of respondent. Figure 7 shows the extent to which each theme was identified as a main barrier by each of the three respondent categories.

Lack of governance, lack of social participation and ineffective integration were the only themes identified as major barriers by all three categories of respondents. The extent to which these issues were identified as major barriers to integration across initiatives varied between respondents from the different categories. It is noticeable that the lack of governance and ineffective integration were accorded considerably higher importance by respondents from local government compared to respondents from civil society and experts.

3 The experts were based on 2 criteria: 1) time frame working with local planning initiatives (minimum 5 years), and 2) participation at least in one phase of the process of implementation. 


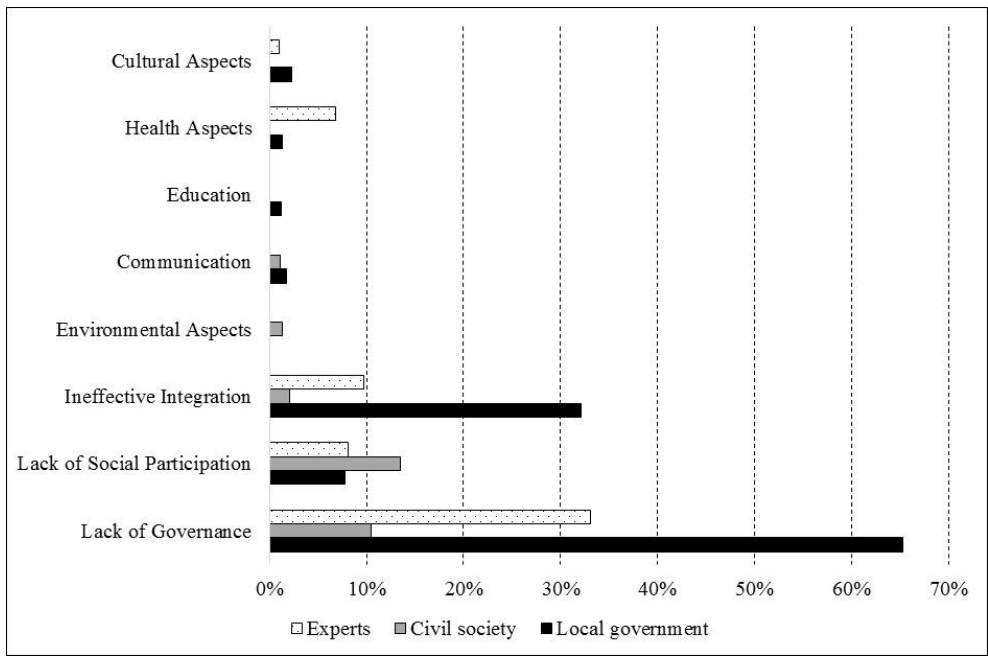

Figure 7: Percentage of each category of respondents who identified a particular theme as a major barrier to effective integration of local planning initiatives.

Respondents from civil society organizations cited a lack of social participation most frequently as a major barrier. For the experts, lack of governance was the most frequently cited barrier. Environmental aspects, communication and legal aspects, education and health-related aspects also emerged as barriers, but were accorded less importance and were not universally recognized by all three categories of respondents.

Regarding enablers of successful integration across initiatives, seven main themes were identified by all categories of respondents, as shown in figure 8. Four themes were identified as enablers by all categories of respondent: good governance, effective social participation, successful integration, and communication. Local government respondents cited good governance most frequently as an important enabler of successful integration of planning initiatives. Good governance was also recognised as being important by experts and civil society respondents.

There is considerable divergence across respondent categories regarding the frequency with which effective social participation was cited as an important enabler. For respondents from civil society organizations and experts, social participation was the most frequently cited important enabler of success, whereas local government respondents cited good governance as an important enabler considerably more frequently. Given their low level of recognition of the importance of social participation, it seems reasonable to conclude that local government representatives might consider that local initiatives could have achieved better results if problems related to the lack of governance could had been overcome without the need for enhanced social participation. Successful integration of initiatives was recognised as an important enabler of success across all categories of respondents.

Environmental aspects, communication, education and legal aspects were also cited as enabling factors, but much less frequently, and were not universally recognised by all respondent categories. 


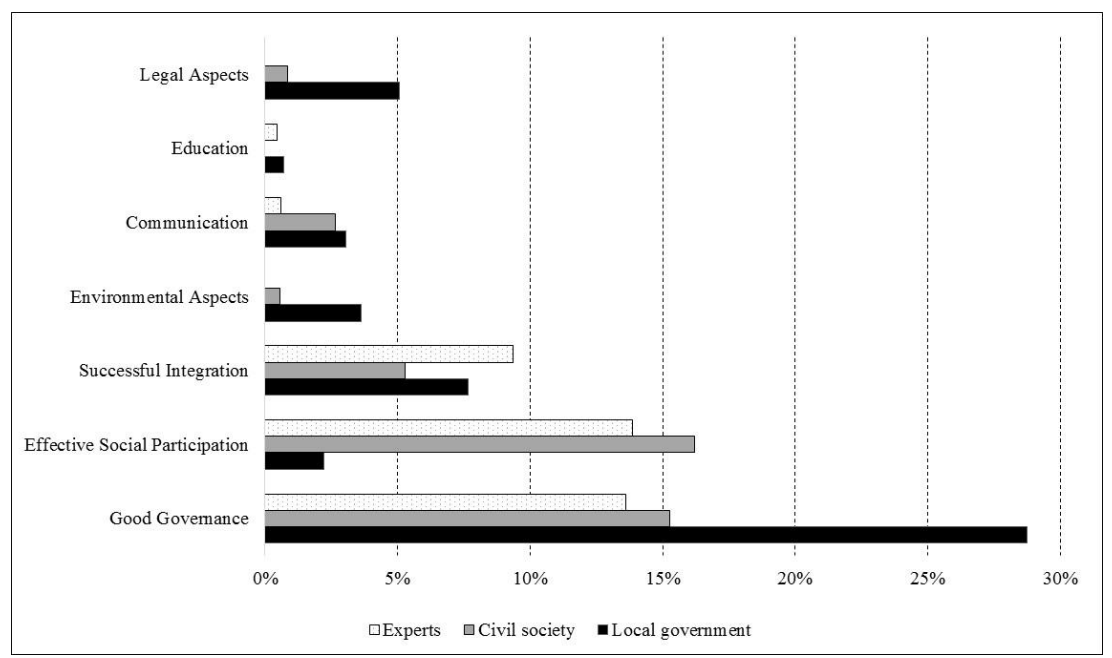

Figure 8:

Percentage of main themes identified as enablers of successful integration of local planning initiatives by experts, local government representatives, and civil society representatives.

\subsection{Implementation of SDG 11 at Local Levels}

Regarding forthcoming implementation of SDG 11 at local levels, the results reflect the perceptions of 30 participants in total, comprising local government representatives, experts and NGOs. Nine key themes were identified as the most important challenges. Figure 9 shows the percentage of interview respondents who identified each theme as important for facilitating successful delivery of SDG11.

'Integration and partnerships' was cited most frequently by stakeholders as an important priority for successful implementation of SDG11. Apart from the political situation in Brazil (P8) and the 'broad and unrealistic Agenda' (P9), it is clear that all the other themes that were cited as priorities for successful implementation of SDG11 are closely related to themes which had been mentioned previously as barriers to implementation of previous and current local development initiatives. This result highlights how important it is to learn from previous experience, and also how useful it could be to overcome repetitive implementation challenges at local level.

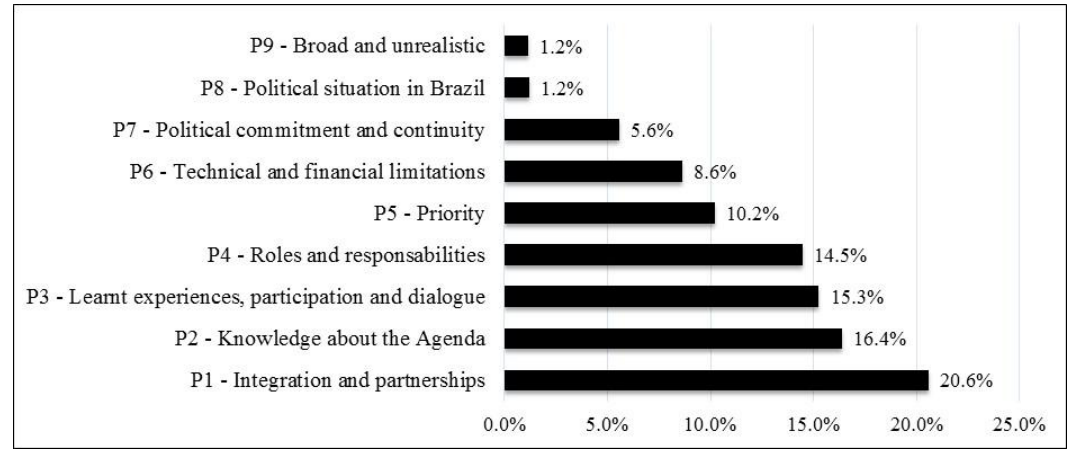

Figure 9: Key themes identified as challenges for the implementation of SDG 11 at local levels based on learned experiences, ranked from most frequently cited P1 to least frequently cited $\mathrm{P} 9$.

The relative frequency with which respondents identified potential challenges to implementation of SDG 11 was used as a starting point for designing a simple framework to facilitate forthcoming implementation of SDG 11 at local levels. Results from the qualitative analysis show that successful incorporation of learned experiences 
regarding barriers and enablers of previous local planning initiatives is widely acknowledged as a priority for successful implementation of SDG 11. The first priority for action in the proposed implementation framework (figure 10) therefore aims to enhance local policies and establish appropriate partnerships. The qualitative analysis in this study suggests that, in this action priority, integration between local governmental agencies, and the establishment of strategic partnerships between distinct sectors are likely to be vital for the success of SDG 11. Two other priorities for action are also suggested as well as enhancing local policies: to enhance understanding of the Agenda, and to strengthen governance. In this study a lack of knowledge about SDGs was evident at local levels. This suggests that it is necessary to keep local governments and citizens well educated and informed about all dimensions of SDG 11. Regarding the priority to strengthen governance, Agenda prioritization and clear definition of roles and responsibilities are critical issues for successful implementation of SDG 11 at local levels. Technical capacity (e.g. staff knowledge, training and experience) and financial limitations, and a lack of political commitment and continuity, were identified by stakeholders as important issues that can hinder implementation of SDGs at local level. Besides that, although the political situation in Brazil and the breadth of the Agenda lie outside the remit of local governments, these issues can potentially affect municipalities and act as negative influences on successful implementation of the Agenda.

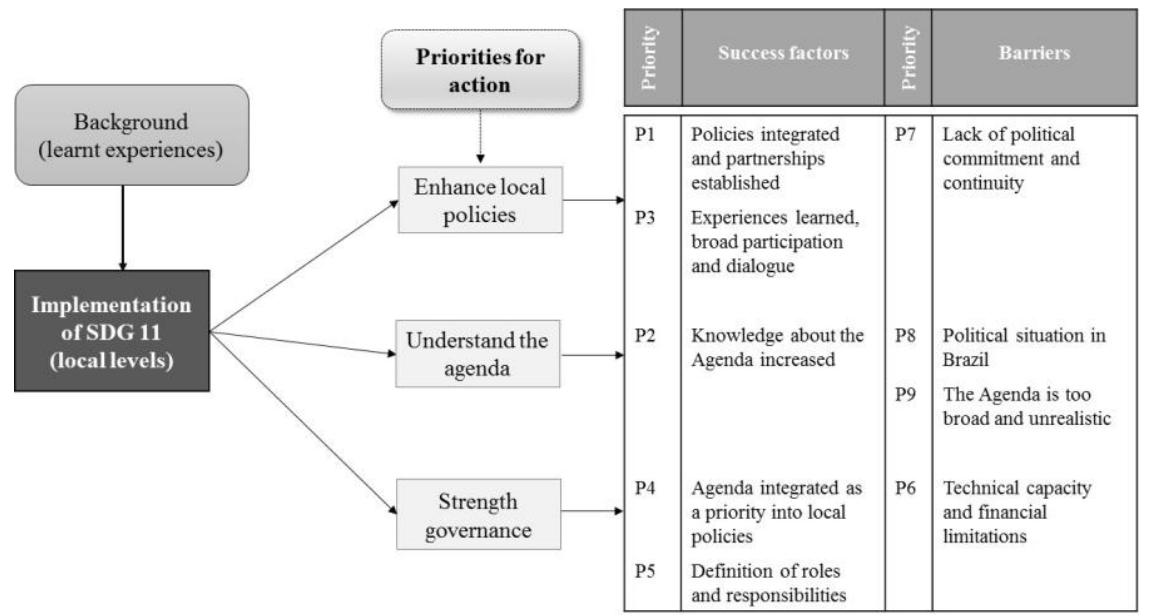

Figure 10: Proposal action framework to enhance implementation of SDG 11 at local levels, drawing on lessons learned from implementing earlier sustainable development initiatives at a local level.

\section{Conclusion}

The action framework proposed in this study can be helpful for enhancing and facilitating implementation of SDG 11 in Brazilian municipalities. Many lessons have been learned from previous Brazilian experience with local-level implementation of sustainable development initiatives. However, it is important to consider whether the very considerable differences between municipalities in Brazil can limit the applicability of the action framework proposed in this research. 
These lessons should be considered in preparing for implementation of SDGs at the local level. Nevertheless, so far, these lessons do not appear to have been considered by local authorities. Quantitative research in this study suggests that an integrated approach for planning initiatives has a positive effect on the development of health, educational and particularly environmental policy. Also, in terms of social participation, this study conclusively identified an associated improvement in the participatory process at local level. The main advances were related more to participation through the Local Councils. Nevertheless, responses obtained from stakeholders within and outside local government suggest that there is still a significant democratic deficit in participation that needs to be corrected. Although local councils allow greater interaction between civil society and local government, Councils will not be able to rectify this deficit alone, but could perhaps lead the process. So, it will be necessary to expand and establish new participatory opportunities, especially by opening pathways for the direct involvement of civil society organizations in implementation processes and follow-up actions. The establishment of social observatories for example could be adopted as an approach for monitoring the implementation of SDGs at local levels.

A major limitation of this research was the lack of access to systematic data collection and reporting of Brazilian municipalities. There is an urgent need to structure a data collection and recording system to provide a baseline from which future changes can be evaluated. Without a well-established baseline it will be very difficult to evaluate the effectiveness of SDG implementations in Brazil. Unfortunately, although an integrated and well-structured data system is essential for this purpose, it remains far from the reality in most Brazilian cities. The research has emphasised that local governments need to provide comprehensive and accessible information to enable citizens to monitor the results of public policies. Using statistical data and survey-derived quantitative responses in combination with a qualitative analysis of societal opinions appears to be an effective research approach for producing a more legitimate evaluation of sustainable development performance. It is recommended that SDG 11 evaluations should be designed based on mixed methods to provide a more inclusive and effective assessment. The action framework designed in this research presents an achievable set of three action areas and nine action priorities that are critical to the implementation of SDG 11 at local levels. This framework provides a basis for the implementation of SDG 11 at local levels. This research has mapped the priorities for action and related these priorities to barriers and success factors. This action framework can also provide insights to enhance the implementation of SDG 11 in practice.

The qualitative research indicated that there is potential for SDG 11 to become a referential goal to enhance sustainability in Brazilian municipalities. SDG 11 also creates an opportunity to influence local governments to think and act on developing sustainable cities. Also, despite some scepticism regarding the ability to implement SDGs at local levels, an appropriate evaluation and action framework provides a consistent vehicle for demonstrating the improvements that are being made; as such it could motivate local governments to improve their performance in SDG implementation and service delivery. 


\section{Acknowledgements}

We would like to acknowledge and thank all participants for their valuable and significant contribution to this work.

\section{References}

Arslan, T. V; Durak, S; Aytac, D. O. (2016). Natural Resources Forum 40, 180-202. DOI: 10.1111/14778947.12115.

Corbett, J; Mellouli, S. (2017). Winning the SDG battle in cities: how an integrated information ecosystem can contribute to the achievement of the 2030 sustainable development goals. Info Systems J. doi: 10.1111 isj.12138.

Glaser, B.G; Strauss, A.L. (1967). Discovery of grounded theory: Strategies for qualitative research. Chicago: Aldine.

Green, G. (2012). Intersectoral Planning for City Health Development. Journal of Urban Health: Bulletin of the New York Academy of Medicine, Vol. 89, No. 2.

Hall, R.P; Ranganathan, S; Kumar G. C., R. (2017). A General Micro-Level Modeling Approach to Analyzing Interconnected SDGs: Achieving SDG 6 and More through Multiple-Use Water Services (MUS). Sustainability 9, 314; doi:10.3390/su9020314

Lichfield N.; Kettle P.; Whitbread M. (1975). Evaluation in the Planning Process. Urban and Regional Planning Series, Vol. 10.

Mcidades. Ministerio das Cidades. (2004). Plano Diretor Participativo: guia para a elaboração pelos municípios e cidadãos. Confea.

NVIVO: NVivo Qualitative Data Analysis Software; QSR International Pty Ltd. Version Strauss, A; Corbin, J. (1998). Basics of qualitative research (2nd ed.). Newbury Park, CA: Sage.

Patel, Z; Greyling, S; Simon, D; Arfvidsson, H; Moodley, N; Primo N; Wright, C. (2017). Local responses to global sustainability agendas: learning from experimenting with the urban sustainable development goal in Cape Town. Sustain Sci 12:785-797 DOI 10.1007/s11625-017-0500-y

Satterthwaite, D. (2016). A new urban agenda? Environment \& Urbanization. International Institute for Environment and Development (IIED). Vol 28(1): 3-12. DOI: 10.1177/0956247816637501

SG-PR. Secretaria-Geral da Presidencia da Republica. (2009). Guia para a Municipalização dos Objetivos do Milênio - Referências para a adaptação de indicadores e metas à realidade local/ Agenda Pública Agência de Ana 'lise e Cooperação em Poli 'ticas Pu 'blicas, São Paulo. Available from: http://www.odmbrasil.gov.br/arquivos/guia-de-municipalizacao.

SPSS: IBM Corp. Released 2012. IBM SPSS Statistics for Windows, Version 21.0. Armonk, NY: IBM Corp.

Strauss, A; Corbin, J. (1998). Basics of qualitative research: Techniques and procedures for developing grounded theory (2nd ed.). Thousand Oaks, CA: Sage.

UNDP. United Nation Development Program. (2016). Roadmap for localizing the SDGs: Implementation and Monitoring at Subnational level. Available from: https://www.uclg.org/sites/default/files/roadmap_for_localizing_the_sdgs_0.pdf

United Nations. Department of Economic and Social Affairs Division for Sustainable Development. (2012). Sustainable Development in the 21st century (SD21). Review of implementation of Agenda 21 and the Rio Principles. Synthesis. http://www.uncsd2012.org/content/documents/194Synthesis $\% 20$ Agenda $\% 2021 \% 20$ and $\% 20$ Rio $\% 20$ principles.pdf

WHO. Regional Office for the Western Pacific. (2000). Regional Guidelines for Developing a Healthy Cities Projects. Available from: http://www.wpro.who.int/health_promotion/documents/regional _guidelines_for_developing_a_healthy_cities_project.PDF. 